Cristiane Caldeira 1

Rita de Cassia Oliveira da Costa Mattos 1 Armando Meyer 1 Josino Costa Moreira 1

\section{Limites de aplicabilidade da determinação do ácido $\Delta$-aminolevulínico urinário como teste screening na avaliação da intoxicação profissional pelo chumbo}

\author{
Applicability limits of urinary $\Delta$-aminolevulinic \\ acid as a screening test to evaluate professional \\ intoxication by lead
}

\footnotetext{
1 Centro de Estudos da Saúde do Trabalhador e Ecologia Humana, Escola Nacional de Saúde Pública,

Fundação Oswaldo Cruz. Rua Leopoldo Bulhões 1480 Manguinhos, Rio de Janeiro, RJ, 21045-900, Brasil.
}

\begin{abstract}
The relationship between concentrations of ALA-U and Pb-S for two groups of workers is reported. The first group consisted of workers from a telephone company, and the second, of workers from battery factories with average $\mathrm{Pb}$-S equivalent to $17,3 \mu \mathrm{g} / \mathrm{dl}(6,2-39,4)$ and 61,5 $\mu \mathrm{g} / \mathrm{dl}$ (41,1-91,0), respectively. The aim of this study was to evaluate the utilization of ALA-U levels as a screening test for different levels of lead in blood by means of High Performance Liquid Chromatography (HPLC) and of spectrophotometry. A significant correlation was found between measured ALA and levels of blood lead $(R=0,739$ - first group; $R=0,902$ - second group; $p<0,001)$. The validity of ALA-U test to evaluate different levels of lead in blood was also studied. By using ALA levels of $3 \mathrm{mg} / \mathrm{g}$ creatinine as a threshold to detect levels of lead in blood equal to or higher than $20 \mu \mathrm{g} / \mathrm{dl}$, the test results, for the workers in the first group, showed sensitivity of $92 \%$ and specificity of $90 \%$. In both groups, the false positives as well as the false negatives were lower than 10\%, which was enough to validate the test.
\end{abstract}

Key words Lead Poisoning; Aminolevulinic Acid; Occupational Exposure; Occupational Health

Resumo A aplicabilidade da determinação da concentração do ácido $\Delta$-aminolevulínico urinário (ALA-U) no controle da saúde de trabalhadores expostos ocupacionalmente ao chumbo foi realizada em dois grupos de trabalhadores. Os valores médios de Pb-S para os dois grupos foram 17,3 $\mu \mathrm{g} / \mathrm{dl}(6,2-39,4)$ e 61,5 $\mu \mathrm{g} / \mathrm{dl}$ (41,1-91,0), respectivamente. As concentrações de ALA-U foram determinadas por HPLC e por espectrofotometria. Uma correlação significativa $(p<0,001)$ foi obtida, relacionando-se os valores de ALA/HPLC com os de Pb-S $(R=0,739$ para o primeiro grupo; $R=0,902$ para o segundo grupo). Também foi avaliado o teste de ALA-U como indicador de diferentes concentrações de chumbo no sangue. Adotando-se a concentração de ALA-U de $3 \mathrm{mg} / \mathrm{g}$ de creatinina como limite para detectar concentrações de Pb-S iguais ou maiores que $20 \mu \mathrm{g} / \mathrm{dl}$, o teste mostrou uma sensibilidade de 92\% e uma especificidade de 90\%. Em ambos os grupos estudados, tanto o número de falsos positivos como o de falsos negativos foram inferiores a 10\%, sendo, portanto, suficientes para validar o teste.

Palavras-chave Intoxicação por Chumbo; Ácido Aminolevulínico; Exposição Ocupacional; Saúde Ocupacional 


\section{Introdução}

O chumbo é um elemento especialmente importante em saúde ocupacional, devido a sua ampla utilização em processos industriais e sua toxicidade aguda e crônica. Por ser altamente resistente a corrosão atmosférica e à ação de ácidos, especialmente o sulfúrico, este metal é muito utilizado em construções, na fabricação de baterias chumbo-ácidas, na fabricação de produtos químicos, tubos, conexões e como envoltório de cabos telefônicos e outros. Desta forma, casos de intoxicação ocupacional causados pelo chumbo são bastante comuns, sendo denominados intoxicação profissional pelo chumbo (IPCH). Apesar da carência de dados brasileiros sistematizados, alguns estudos isolados têm demonstrado uma alta prevalência destes casos em nosso país. A intoxicação profissional pelo chumbo, por ser uma doença grave, incapacitante e de grande incidência ocupacional, é considerada um problema de saúde pública, sendo passível de prevenção através da adoção de processos de higiene industrial e equipamentos de proteção coletiva (ATSDR, 1990).

A toxicidade do chumbo se manifesta principalmente em quatro sistemas: gastrointestinal, renal, nervoso e hematopoiético, sendo este último de grande importância no monitoramento biológico à exposição a este metal. $\mathrm{O}$ principal efeito do chumbo neste sistema é a redução dos níveis do grupo prostético heme, causado pela inibição de algumas enzimas utilizadas na síntese da hemoglobina, devido a ligação do metal à enzima ácido $\delta$-aminolevulínico desidratase (ALA-D), causando o acúmulo do ácido delta aminolevulínico (ALA) no sangue e na urina (Alessio \& Foá, 1983; ATSDR, 1990; Landrigan, 1989; Paolielo et al., 1993). Deste modo, a determinação da concentração do ácido $\Delta$-aminolevulínico urinário (ALA-U) tem sido proposta para o monitoramento da exposição ocupacional ao chumbo, paralelamente aos níveis do metal no sangue (Pb-S).

Alguns autores tem sugerido a utilização do ALA-U como teste screening para se estudar a intoxicação humana pelo chumbo, por ser um método não-invasivo, causando o mínimo de desconforto, e por utilizar uma matriz biológica de fácil coleta e análise. A utilização de métodos mais sensíveis para a determinação deste metabólito em fluidos biológicos tem possibilitado a detecção cada vez mais precoce dos casos de intoxicação pelo chumbo onde ainda não são observados sinais e/ou sintomas da intoxicação. A utilização da Cromatografia Líquida de Alta Performance (HPLC) como método analítico, ao invés do método espectrofotométrico, comumente utilizado no Brasil, representa um sensível avanço nesta determinação, por incorporar uma etapa prévia de separação cromatográfica e, assim, diminuir a interferência causada por outros compostos como aminoacetonas, presentes na urina (Hennekens \& Buring, 1987; Simmonds et al., 1995; Tabuchi et al., 1989).

Estudos realizados com algumas atividades profissionais e em ambientes de trabalho no Brasil têm demonstrado valores elevados de chumbo no ar-ambiente de locais onde se utiliza este metal (acima de $0,1 \mathrm{mg} / \mathrm{m} 3$ ), resultando em um grande percentual de trabalhadores contaminados com níveis sangüíneos acima de $25 \mu \mathrm{g} / \mathrm{dl}$ (Araujo et al., 1999). É importante ressaltar que problemas de saúde têm sido associados mesmo a estes níveis de chumbo no sangue (ATSDR, 1990).

\section{Material e métodos}

Os níveis de $\mathrm{Pb}$-S e de ALA-U foram determinados em amostras de sangue e urina de 82 trabalhadores expostos ao chumbo em dois tipos de atividades, dos quais 65 trabalhavam como cabistas de uma companhia telefônica e 17 em fábricas de baterias, localizadas na região do Grande Rio. Dentre os cabistas, a população estudada é composta de homens adultos, com idade na faixa de 35 a 56 anos $(x=46)$, com tempo de serviço na empresa variando entre cinco e 30 anos $(x=20)$, sendo que $63,2 \%$ dos trabalhadores ocupam a mesma função há mais de dez anos. A população das fábricas de baterias estudada é composta por homens adultos que pertenciam ao setor de montagem (área considerada de maior risco), com idade na faixa de 24 a 28 anos $(x=26)$, com tempo de serviço variando de quatro semanas a seis anos.

Os níveis de ALA-U foram determinados por dois métodos: HPLC e espectrofotometria UV.

Nas análises por HPLC, o método utilizado foi o recomendado por Ogata \& Taguchi (1987) modificado, que consiste no uso de metilacetoacetato na etapa de condensação e formação do ALA-pirrol e do N-butanol para diminuir o número de interferentes encontrados na urina, impedindo a formação de outros pirróis. Todas as determinações foram realizadas com o uso de um cromatógrafo, HP modelo 1090, equipado com válvula de injeção Rheodine com detetor espectrofotométrico no ultra-violeta/visível tipo Diode-array.

O seguinte procedimento foi adotado na realização destas análises: a $1 \mathrm{ml}$ de urina foram 
adicionados 0,2 ml de solução de ácido acético (60\%) e $2 \mathrm{ml}$ de N-butanol. A separação das fases orgânica e aquosa foi realizada por centrifugação por três minutos a $2.000 \mathrm{rpm}$. À fase aquosa foram acrescentados $0,5 \mathrm{ml}$ de solução tampão fosfato $(\mathrm{pH}=6,8)$ e $0,1 \mathrm{ml}$ de metilacetoacetato. A mistura foi aquecida a $100 \circ \mathrm{C}$ por dez minutos e após resfriamento foi injetada no HPLC. Curvas analíticas foram preparadas utilizando-se soluções-padrão nas concentrações de 2,5, 5, 10, 20 e 40mg/l, preparadas a partir de uma solução-padrão estoque de $1 \mathrm{~g} / \mathrm{l}$ do cloridrato de ALA.

As seguintes condições operacionais foram utilizadas: fase móvel: acetonitrila/solução de KH2PO4 50 mM a pH 2,5 ajustado com ácido fosfórico (proporção de 20:80); velocidade de fluxo: 1,0 ml/minuto; coluna: fase reversa C 18 $(15 \mathrm{~cm} / 4,6 \mathrm{~mm})$; detecção UV a $260 \mathrm{~nm}$; temperatura $40 \circ \mathrm{C}$; loop de $50 \mu \mathrm{l}$.

Estudos de recuperação, sensibilidade e reprodutibilidade do procedimento analítico foram realizados, obtendo-se valores médios de $90+/-2 \%$ de recuperação trabalhando-se com amostras de urina às quais se adicionou ALA até obter-se concentrações de 2, 5, 10 e $40 \mathrm{mg} / \mathrm{l}$. As reprodutibilidade inter e intra ensaios foram determinadas utilizando-se as mesmas concentrações, e os coeficientes de variação obtidos foram sempre inferiores a $10 \%$.

O limite de detecção do método utilizado

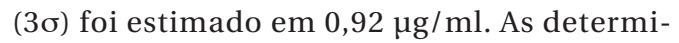
nações espectrofotométricas de ALA-U foram realizadas de acordo com o método descrito por Tomokumi \& Ogata (1980, apud Tabuchi et al., 1989), que se baseia na formação de um composto colorido na reação entre o ALA-pirrol e o reativo de Erlich modificado, sendo a absorvância resultante medida a 532nm. Para isto, foi utilizado um espectrofotômetro Shimadzu $160 \mathrm{~A}$.

Foram realizadas análises dos coeficientes de variação, assim como estudos de recuperação e reprodutibilidade. Obteve-se, em média, $89 \%$ de recuperação para as concentrações de 2,5, 10 e $40 \mathrm{mg} / \mathrm{l}$. As reprodutibilidades inter e intra ensaios foram determinadas para as mesmas concentrações, com coeficientes de variação inferiores a $12 \%$. O limite de detecção do método foi estimado em $2 \mu \mathrm{g} / \mathrm{ml}$.

\section{Resultados e discussão}

A avaliação e interpretação dos resultados teve como base os valores de referência obtidos no estudo da população não exposta da cidade de Londrina, Paraná (Okayma et al., 1990), que são: $\mathrm{Pb}-\mathrm{S}$ até $14,7 \mu \mathrm{g} / \mathrm{dl}$ e ALA-U igual a 5,3 $\mathrm{mg} / \mathrm{g}$ de creatinina $(\mathrm{mg} / \mathrm{g} \mathrm{cn})$.

Os resultados obtidos no estudo comparativo entre os métodos, cromatográfico (HPLC) e espectrofotométrico, utilizados neste trabalho é mostrado na Figura 1. Como pode ser observado, o método cromatográfico demonstrou maior especificidade, principalmente em baixas concentrações de $\mathrm{Pb}$-S, categorizando uma porcentagem maior de indivíduos abaixo de $4,5 \mathrm{mg} / \mathrm{g}$ cn de ALA-U, adotado como ponto de corte.

A correlação entre os resultados obtidos pelos dois métodos foi boa $(r=0,968, p=0,001)$, como pode ser observado na Figura 2.

Tanto as concentrações de $\mathrm{Pb}$-S quanto as de ALA-U determinadas neste estudo distribuíram-se em uma ampla faixa (Pb-S entre 6,291,0 $\mathrm{g} / \mathrm{dl}$; ALA-U entre 1,0-43,9 mg/g/cn e 2,0$35,9 \mathrm{mg} / \mathrm{g} \mathrm{cn}$, para os dois grupos estudados).

Na Tabela 1 são apresentados os resultados das concentrações de Pb-S e de ALA-U, organizados em grupos, utilizando-se os resultados obtidos pelos dois métodos. O primeiro grupo foi composto pelos trabalhadores da companhia telefônica com concentrações de $\mathrm{Pb}-\mathrm{S}$ próximas ao valor utilizado como de referência (14,3 ug/dl). A média dos valores do ALA para este grupo foi de $2,8 \mathrm{mg} / \mathrm{g} \mathrm{cn}$, quando utilizados os dados obtidos por HPLC. No segundo grupo, foram incluídos os trabalhadores das fábricas de baterias, nos quais o valor médio de $\mathrm{Pb}-\mathrm{S}$ e os de ALA-U foram mais elevados. Estes

Figura 1

Relação entre a distribuição percentual dos trabalhadores com níveis de ALA-U abaixo de $4,5 \mathrm{mg} / \mathrm{g}$ cn e os valores de $\mathrm{Pb}-\mathrm{S}$.

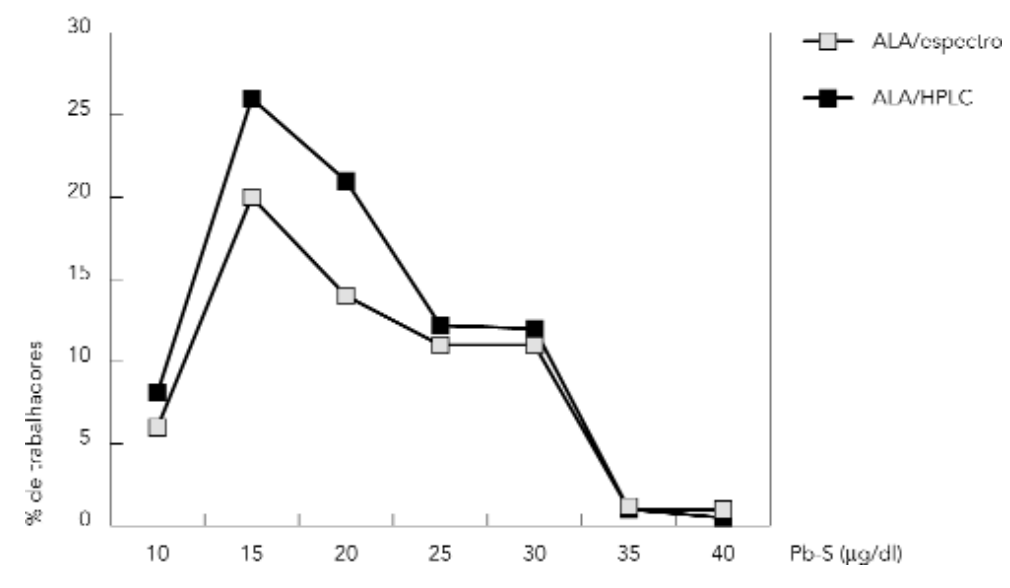


Tabela 1

Comparação dos valores de ALA-U obtidos pelos dois métodos em trabalhadores da companhia telefônica e de fábrica de baterias.

\begin{tabular}{|c|c|c|c|}
\hline Grupos & $\begin{array}{c}\text { ALA/espectro } \\
\mathrm{mg} / \mathrm{g} \mathrm{cn}\end{array}$ & $\begin{array}{c}\text { ALA/HPLC } \\
\mathrm{mg} / \mathrm{g} \mathrm{cn}\end{array}$ & $\begin{array}{l}\mathrm{Pb}-\mathrm{S} \\
\mu \mathrm{g} / \mathrm{dl}\end{array}$ \\
\hline \multicolumn{4}{|l|}{ Grupo1/cabistas } \\
\hline Média (IC 95\%) & $3,01 \pm 0,37$ & $2,86 \pm 0,48$ & $17,3 \pm 2,0$ \\
\hline Desvio padrão & 1,5 & 1,7 & 8,2 \\
\hline \multirow[t]{2}{*}{ Faixa } & $1,1-6,8$ & $1,0-10,9$ & $6,2-39,3$ \\
\hline & $n=65$ & $n=48$ & $\mathrm{n}=65$ \\
\hline \multicolumn{4}{|l|}{ Grupo2/fábrica } \\
\hline Média (IC 95\%) & $24,7 \pm 6,57$ & $14,7 \pm 2,12$ & $63,8 \pm 8,37$ \\
\hline Desvio padrão & 9,21 & 20,15 & 16,27 \\
\hline \multirow[t]{2}{*}{ Faixa } & $3,3-35,5$ & $2,2-43,9$ & $41,1-91,0$ \\
\hline & ${ }^{*} \mathrm{n}=10$ & $n=17$ & $\mathrm{n}=17$ \\
\hline
\end{tabular}

* Nesse grupo estão incluídos apenas os trabalhadores de uma das fábricas de baterias.
Esses resultados demonstram que para concentrações de $\mathrm{Pb}$-S maiores que 6,2 $\mu \mathrm{g} / \mathrm{dl}$, obteve-se uma boa correlação entre os dois biomarcadores. Resultados descritos na literatura demonstram uma boa correlação entre os valores de ALA-U determinados espectrofotometricamente e os de $\mathrm{Pb}-\mathrm{S}$ apenas em concentrações muito mais elevadas que aquelas aqui descritas (Pb-S >18 $\mu \mathrm{g} / \mathrm{dl}$ ) (Skerfving, 1993). A utilização de métodos analíticos cada vez mais sensíveis e seletivos, como a HPLC, para monitoramento ocupacional permite detectar-se níveis de contaminação, aos quais são observados apenas pequenas alterações metabólicas reversíveis, facilitando, desta forma, a adoção de medidas preventivas (modificações no ambiente de trabalho, mudanças para setores de menor risco ou afastamento do trabalhador de suas atividades), evitando-se maiores danos à saúde dos trabalhadores.

Para os trabalhadores das fábricas de baterias também foi encontrada uma boa correlação ( $r=0,902, p=0,001)$ entre Pb-S e ALA-U para concentrações mais elevadas de $\mathrm{Pb}-\mathrm{S}$ ( $\mathrm{x}=62,5 \mu \mathrm{g} / \mathrm{dl}$ ) (Tomokuni et al., 1988). valor muito superior ao IBMP (Índice Biológico Máximo Permitido) recomendado pela legislação brasileira.

Nos trabalhadores do grupo 1, os valores médios de ALA-U determinados espectrofotometricamente foram 1,5 vezes maiores que os obtidos por HPLC, enquanto que, no grupo 2, esta relação foi de 1,7. Esses dados sugerem a interferência de outros compostos, como, por exemplo, aminoacetonas em todas as faixas de concentração estudadas. Esse fato foi descrito por Ogata \& Tabuchi (1987, apud Létorneau et al., 1988) apenas para baixas concentrações de ALA-U.

A avaliação da correlação entre os logaritmos das concentrações de ALA-U e os níveis de chumbo em sangue, $\mathrm{Pb}-\mathrm{S}$, foi realizada utilizando-se os valores de ALA-U obtidos por HPLC, por ser este o método mais seletivo. Estas correlações foram testadas, separadamente, primeiro nos resultados obtidos dos trabalhadores da companhia telefônica e, depois, nos obtidos dos trabalhadores das fábricas de baterias. Estes testes foram realizados no sentido de verificar-se a especificidade do método, principalmente em níveis baixos de $\mathrm{PbS}$.

Os resultados obtidos serão apresentados separadamente.

Apesar das médias das concentrações de $\mathrm{Pb}-\mathrm{S}$ e ALA-U dos cabistas telefônicos (17,3 $\mu \mathrm{g}$ / dl e 2,8 mg/g cn, respectivamente) estarem próximas ou até abaixo do valor utilizado como de referência $(14,3 \mu \mathrm{g} / \mathrm{dl}$ e 5,4 mg/g cn), foi encontrada uma correlação estatisticamente significativa $(r=0,739, p=0,01)$ entre os biomarcadores estudados (Figura 3 ).

\section{Conclusão}

A utilização do ALA-U como teste screening de avaliação da intoxicação de trabalhadores por chumbo tem como vantagem evitar que indivíduos com baixa carga corpórea do metal sejam submetidos a exames invasivos periódicos, podendo, no caso de exposições leves, reduzir o número de análises em até $70 \%$. As vantagens de um biomarcador que possa ser analisado utilizando-se como matriz biológica a urina são claras. Este tipo de amostra não traz nenhum tipo de desconforto ao trabalhador, apresenta maior facilidade na coleta, armazenamento, transporte e manuseio. A desvantagem causada pela influência da variabilidade inter-individual da urina pode ser contornada, em parte, pela correção das amostras pela creatinina, com os resultados expressos em $\mathrm{mg} / \mathrm{g}$ de creatinina.

Portanto, pode-se sugerir a utilização do ALA-U como indicador biológico útil para selecionar trabalhadores expostos. Alguns cuidados devem ser considerados devido a possibilidade de testes falso-positivos. O fato do resultado do teste se situar na faixa de normalidade, entretanto, não significa saúde, e qualquer sinal ou sintoma apresentado pelo trabalhador não deve ser ignorado. Além da necessidade de exames clínicos e laboratoriais periódicos, avaliações integradas ao monitoramento do ambiente de trabalho são também imprescindíveis no sentido de se garantir a saúde do trabalhador. 


\section{Figura 2}

Dispersão dos logaritmos dos resultados de ALA-U obtidos por HPLC e espectrofotometria UV/visível para todos os trabalhadores estudados.

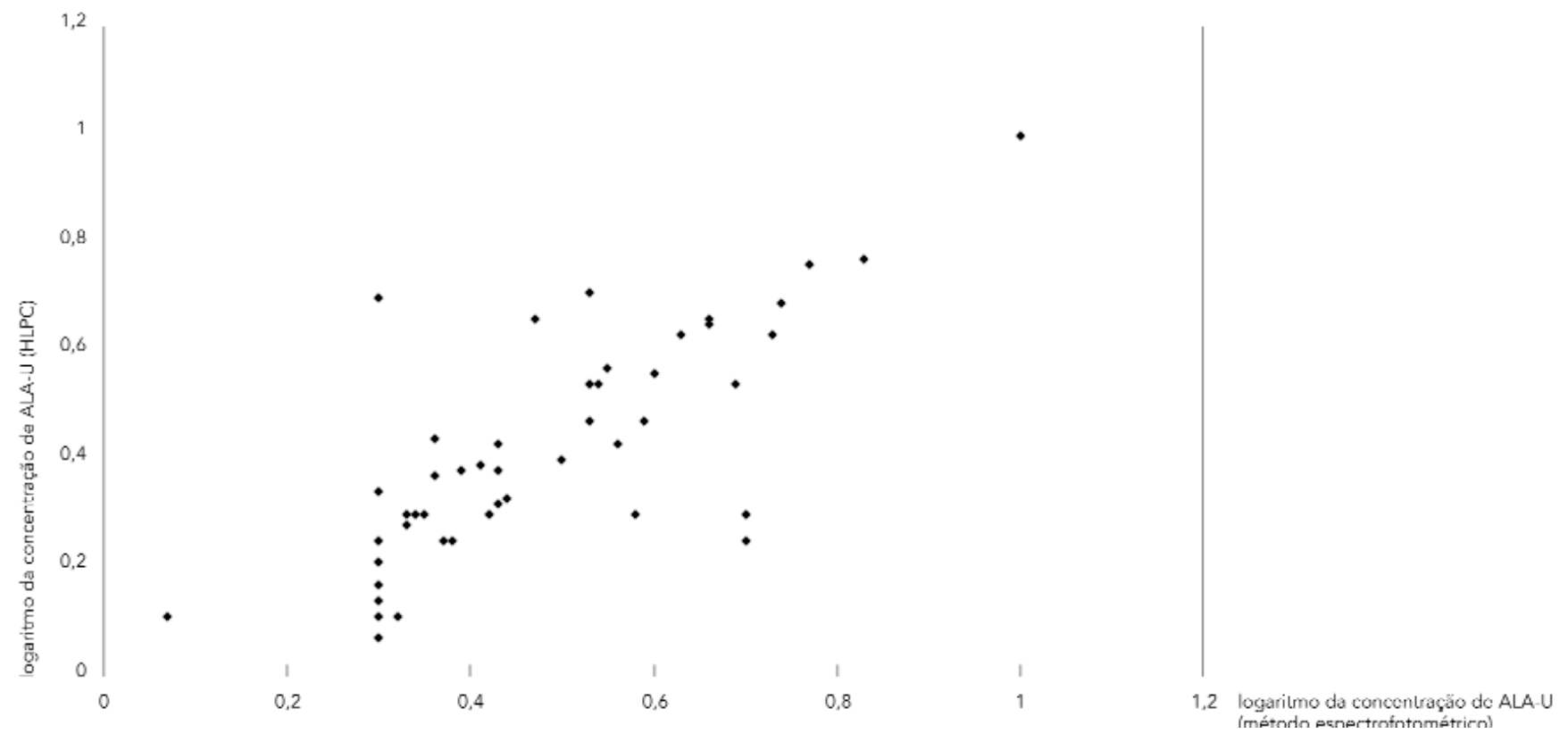

Figura 3

Estudo da correlação entre $\mathrm{Pb}-\mathrm{S}$ e log ALA-U: dispersão dos resultados obtidos para os trabalhadores da companhia telefônica.

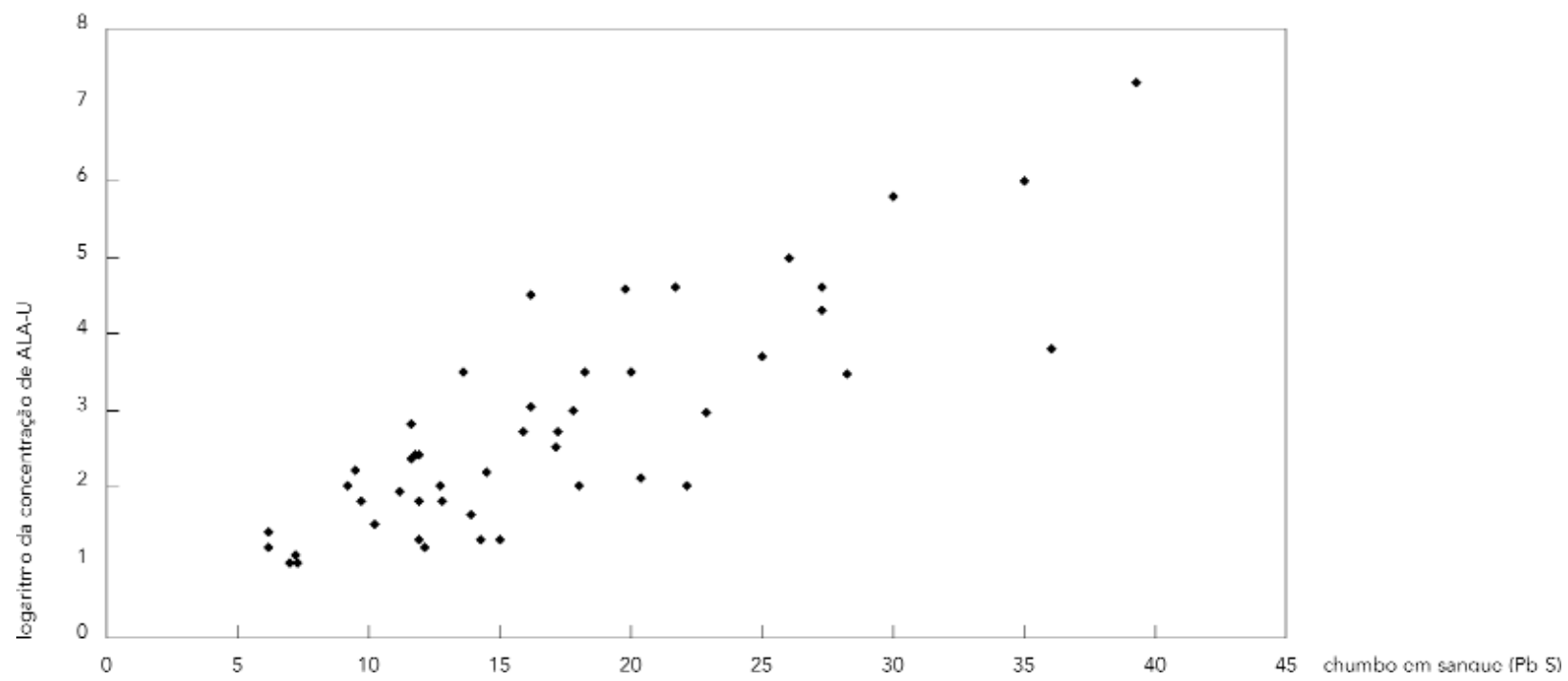




\section{Referências}

ALESSIO, L. \& FOÁ, V., 1983. Lead. In: CCE Monographs on Human Biological Monitoring of Industrial Chemicals Series (L. Alessio, A. Berlin, M. Boni \& R. Roi, eds.), pp. 105-132, Amsterdam: Elsevier Science BV.

ARAUJO, U. C.; PIVETTA, F. R. \& MOREIRA, J. C., 1999. Avaliação da exposição ocupacional ao chumbo: Proposta de uma estratégia de monitoramento para a prevenção de efeitos clínicos e sub-clínicos. Cadernos de Saúde Pública, 15:123-132.

ATSDR (Agency for Toxic Substances and Disease Register), 1990. Toxicological Profile for Lead. Washington, D.C.: ATSDR.

CORDEIRO, R., 1995. Quando Começa o Saturnismo? Tese de Doutorado, São Paulo: Faculdade de Ciências Médicas, Universidade Estadual de Campinas.

HENNEKENS, C. \& BURING, J. E., 1987. Epidemiology in Medicine. Boston/Toronto: Little, Brown and Company.

LANDRIGAN, P. J., 1989. Toxicity of lead at low dose. British Journal of Industrial Medicine, 46:593-596.

LÉTORNEAU, G. G.; PLANTE, R. \& WEBER, J. P., 1988. Blood lead and maximal urinary excretion of $\delta$ aminolevulinic acid. American Industrial Hygiene Association Journal, 49:342-345.

OGATA, M. \& TAGUCHI, T., 1987. High performance liquid chromatographic procedure for quantitative determination of urinary delta-aminolevulinic indices of lead exposure. International Archives of Occupational and Environmental Health, 59: 385-391.

OKAYMA, A.; FUJII, S. \& MIURA, R., 1990. Optimized fluorimetric determination of urinary d-aminolevulinic acid by using pre column derivatization, and identification of the derivative. Clinical Chemistry, 36:1494-1497.
PAOLIELO, M. M. B.; TURINI, C. A.; GUTIERREZ, P. R.; MEZZAROBA, L.; BARBOSA, D. S.; SOUZA, J. L. K.; MANAKA, R. H.; MATSUO, T.; MARTINS, M. B. B.; FIGUEROA, G. V.; TEIXEIRA, J. S.; SIQUEIRA, A. M. M. \& MELLO, S. R. B., 1993. Valores de referência para indicadores biológicos de exposição ao chumbo na população da região urbana de Londrina-PR: Uma proposta metodológica. Revista Brasileira de Toxicologia, 6:144-148.

SKERFVING, S., 1993. Inorganic lead. In: Criteria Documents from the Nordic Expert Group (B. Beije \& P. Lundberg, eds.), pp. 125-238, Lund: Asbete och Hälsa.

SIMMONDS, P. L.; LUKHURST, C. L. \& WOODS, J. S., 1995. Quantitative evaluation of Heme biosynthetic pathway parameters as biomarkers of lowlevel lead exposure in rats. Journal of Toxicology and Environmental Health, 44:351-367.

TABUCHI, T.; OKAYAMA, A.; OGAWA, Y.; MIYAJIMA, K.; HIRATA, M.; YOSHIDA, T.; SUGIMOTO, K. \& MORIMOTO, K., 1989. A new HPLC fluorimetric method to monitor urinary delta aminolevulic acid (ALA-U) levels in workers exposed to lead. International Archives of Occupational and Environmental Health, 61:297-302.

TOMOKUNI, K.; ICHIBA, M.; HIRAI, Y.; SUGIMOTO, K.; YOSHIDA, T. \& HIRATA, M., 1988. Comparation between the fluorimetric HPLC method and the conventional method for determining urinary $\delta$-aminolevulic acid and coproporphirin as indices of lead exposure. International Archives of Occupational and Environmental Health, 61:153156. 